\title{
A 42-year-old MAle With Blurry Vision
}

William H Chong, $M D$, Dorothy Chang, $M D$

\section{Introduction}

Hypertension affects approximately $25 \%$ of the population of the United States. Complications from hypertension include ischemic heart disease and stroke, and rates increase progressively as blood pressure increases. ${ }^{1}$ In some cases, the elevation of blood pressure can be significant and be life threatening. Situations where there is severe elevation of blood pressure and evidence of target organ dysfunction are termed hypertensive emergency. In this report, an unusual cause of hypertensive emergency is presented.

\section{Case Report}

A 42 year-old male with no significant past medical history presented to the emergency department after being seen at the optometrist for a complaint of blurry vision and was found to have markedly elevated blood pressure. He reported wavy vision from his left eye as well as decreased peripheral vision and decreased color vision. He noted 3 episodes of left-sided headaches over the last month, but has otherwise felt well. He reported no chronic medications, and no substance abuse. Family history was significant for a brother with diabetes. Review of systems was negative aside from the above-mentioned visual changes. He reported no chest pain, no shortness of breath, no abdominal complaints, and no urinary complaints. Physical exam was significant for severe hypertension with blood pressure of $245 / 115$. Otherwise his exam was unremarkable. A report from the optometry clinic noted that he had retinal changes consistent with hypertensive emergency (Figure 1a, 1b).

The gentleman was admitted to the intensive care unit for malignant hypertension. His admission labs were significant

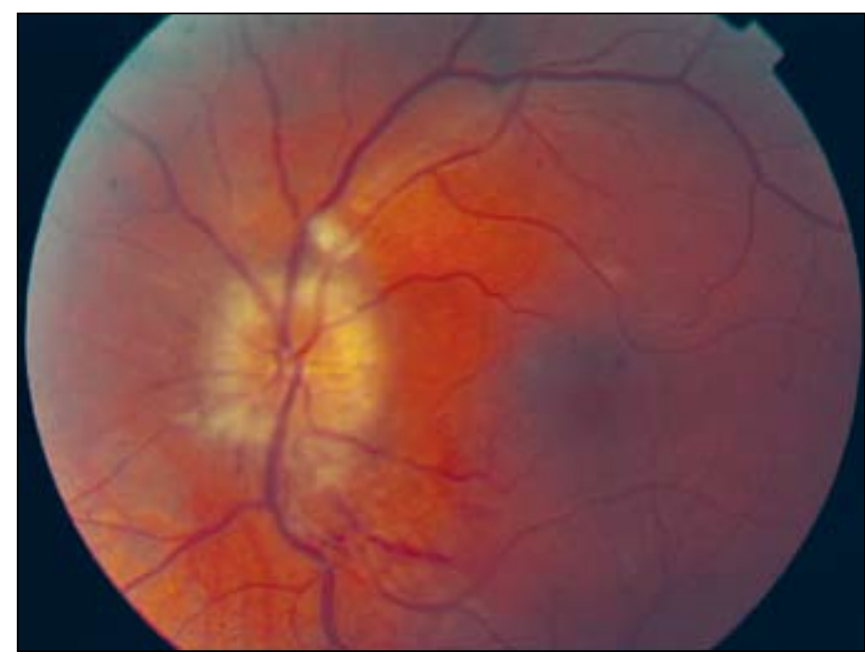

Figure 1. 42 year old male presented to the optometry clinic with complaint of blurry vision and was found to have retinal findings consistent with malignant hypertension. (Images courtesy of Wilmington VAMC) for a creatinine of $3.5 \mathrm{mg} / \mathrm{dL}$. In addition to placing him on a labetalol infusion for blood pressure control, work-up of his renal failure revealed bilateral hydronephrosis and a massively distended bladder rising above the level of the umbilicus (Fig $2 \mathrm{a}, 2 \mathrm{~b})$. Upon placement of a foley catheter and drainage of retained urine, his creatinine began to improve and his blood pressure became more manageable. Urologic evaluation revealed urethral stricture. Subsequent clinical inquiries revealed a distant history of urethral trama as a child which had not been problematic previously. Work-up for other causes of his malignant hypertension were unrevealing. The cause of his hypertensive emergency was attributed to renal failure secondary to urethral stricture. He was placed on oral medications and instructed to perform clean intermittent catheterization to prevent future urinary retention and was discharged home. Outpatient discharge instructions included urology \& nephrology follow-up with a scheduled lumbar spine MRI to evaluate for neurologic causes of urinary retention.

\section{Discussion}

Hypertensive emergencies are acute, life threatening, and usually associated with marked increases in blood pressure, generally above $180 / 120 .{ }^{2}$ Blood pressures elevated to this degree without evidence of acute and progressive dysfunction of target organs is termed hypertensive urgency. Most patients with hypertensive emergency have had previously uncontrolled or unknown chronic hypertension. However, the disorder can present in previously normotensive individuals. ${ }^{3}$ In addition to marked elevation in blood pressure, major clinical manifestations include retinal hemorrhages and exudates, papilledema, and also malignant nephrosclerosis.

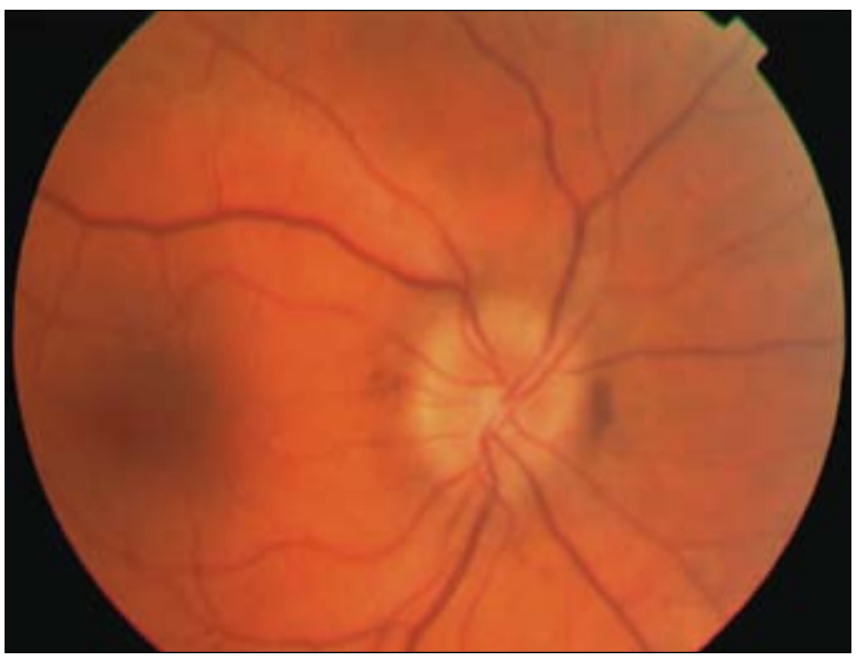

A. Right fundoscopic exam demonstrating a normal exam

B. Left fundoscopic exam demonstrating blurring of optic disc (papilledema) consistent with malignant hypertension. 

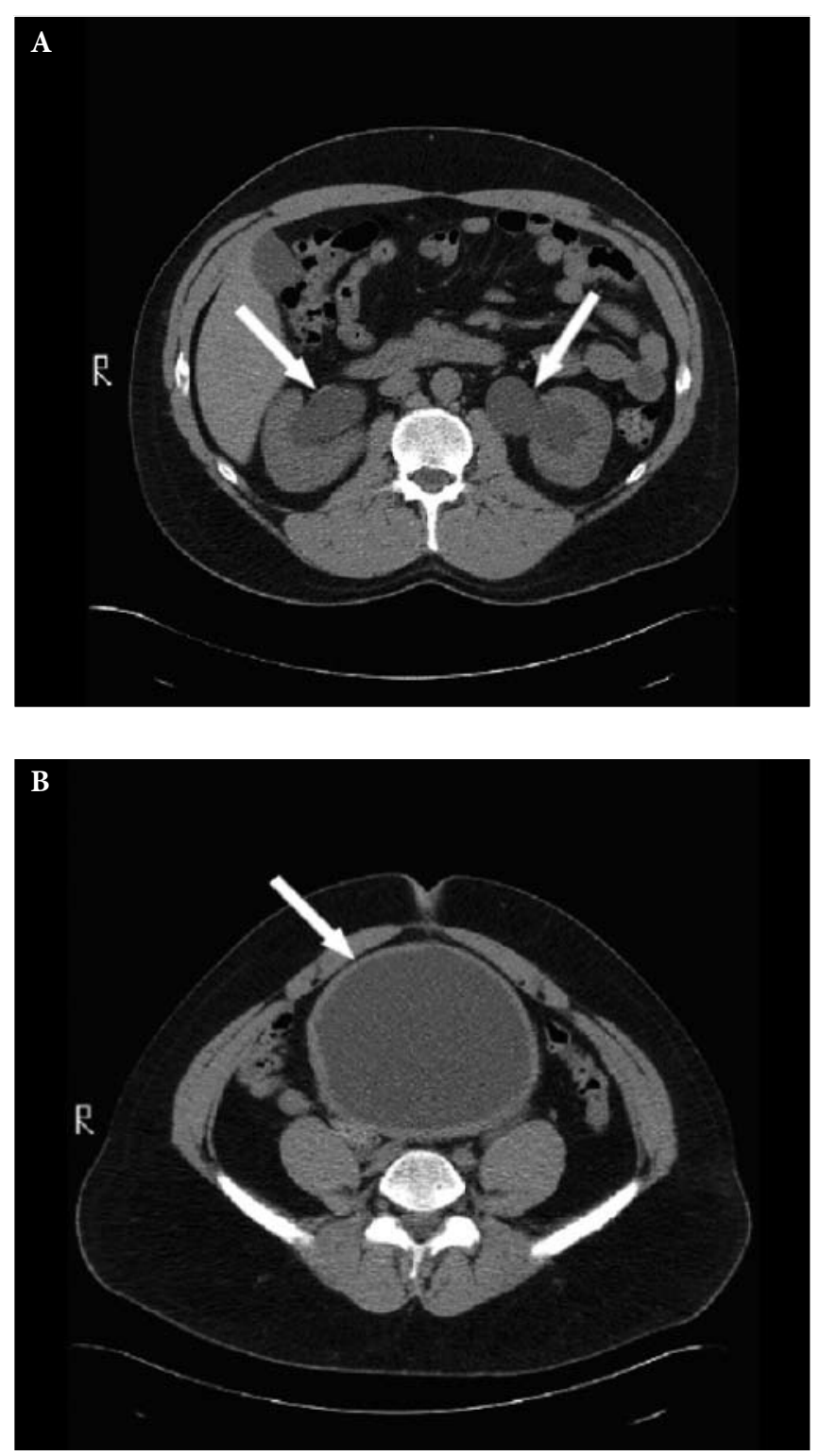

Figure 2. Following admission, pt found to have significant urinary retention with renal failure and malignant hypertension believed to occur secondarily. (Images courtesy of Wilmington VAMC)

A: Computerized tomography of abdomen demonstrating bilateral hydronephrosis (arrows).

B: Massively distended bladder consistent with outflow obstruction at the level of the umbilicus (arrow).
In assessing patients with severe hypertension to determine if hypertensive emergency is present, certain history and physical exam points should be emphasized. Medical history should include previous treatments, illicit drug use, cardiovascular manifestations, neurologic symptoms, and urinary habits. Eliciting information about other medical conditions, such as thyroid disease, Cushing's syndrome, systemic lupus erythematosus, systemic sclerosis, abdominal pain, dyspnea, and most recent menstruation can be extremely helpful. ${ }^{3}$ Physical exam should also assess blood pressure measured in both arms to detect any significant difference. Additionally, assessment of peripheral pulses for absence or delay is of clinical utility. Fundoscopy, cardiac and lung auscultation, and assessment of mental status are also important. Target organ dysfunction can be further revealed by analysis of laboratory studies and the presence of any EKG abnormalities.

In treating hypertensive emergency, the main objective is to reverse end-organ damage, which is accomplished by reducing mean arterial pressure by up to $25 \% .3$ In general, blood pressure should be reduced by $\sim 10 \%$ in the first hour and another $15 \%$ gradually over the next two to three hours. 3 This is best accomplished with a continuous infusion of a short acting, titratable, parenteral anti-hypertensive agent along with constant, intensive patient monitoring.1 Anti-hypertensive drugs of choice include sodium nitroprusside, nitroglycerin, nicardipine, fenoldopam, labetalol, esmolol, hydralazine, and phentolamine. Co-morbidities and the type of target organ dysfunction dictate which anti-hypertensive agent should be selected.

In summary, this is a case of a 42 year-old male initially presenting with blurry vision secondary to hypertensive emergency. It is presumed that the etiology of this hypertensive emergency episode was secondary to renal failure caused by a urethral stricture. Hypertensive emergency is defined by severe hypertension (usually above 180/120) and evidence of target organ dysfunction. In these situations, prompt blood pressure control by no more than $25 \%$ within the first several hours prevents further end organ dysfunction. Numerous titrable, anti-hypertensive agents exist and should be tailored to the patient's clinical history to prevent acute relative hypotension and watershed infarct.

\section{References}

1. McCowan C. Hypertensive emergencies. www.emedicine.com. 2 Aug 2007.

2. Kaplan NM, Rose BD. Hypertensive emergencies: Malignant hypertension and hypertensive encephalopathy. www.uptodate.com, 2007.

3. Feldstein C. Management of hypertensive crises. American Journal of Therapeutics. 2007; 14: 135-139. 